RELIQUIDACIONES

(Informe de Práctica Profesional)

\author{
Propuesta presentada a: \\ Universidad Santo Tomás-Sede Medellín \\ Primer Claustro Universitario de Colombia
}

\author{
Elaborado por: \\ Catherine Rodriguez Cruz \\ Auxiliar Contable \\ Apoyado por: \\ Gloria Inés Sanín Arroyave \\ Presidente Junta Directiva - Tropy Fruis SAS \\ Faber Arley Restrepo \\ Contador público - Tropy Fruits SAS
}

Febrero 11 de 2018

Medellín 
Tabla de contenido

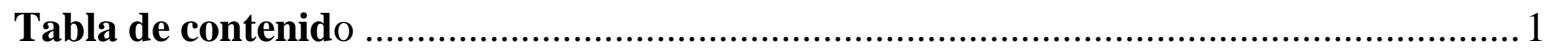

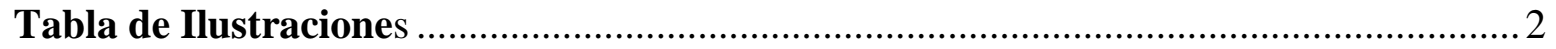

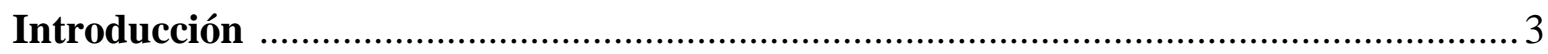

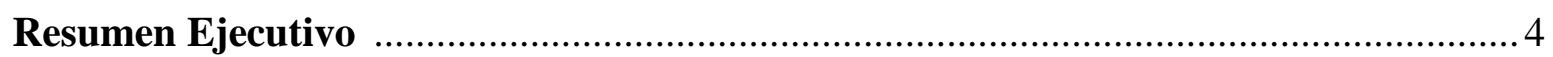

Breve Descripción de la Empresa............................................................................

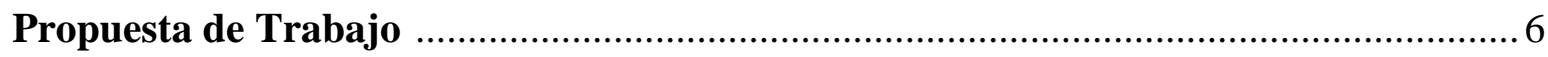

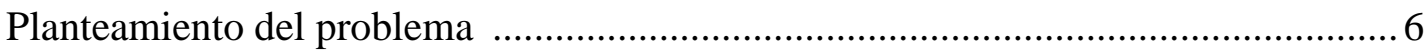

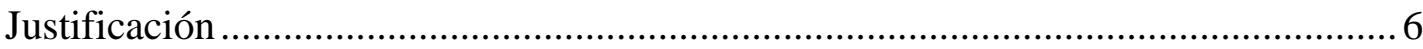

Desarrollo de la Práctica Profesional............................................. 7

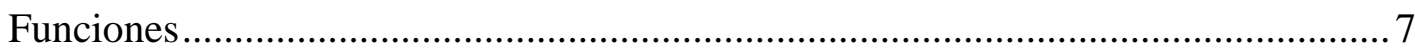

Metodologia Implementada ................................................................................... 8

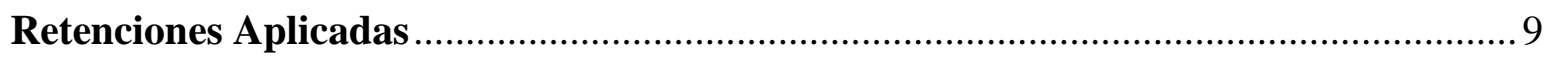

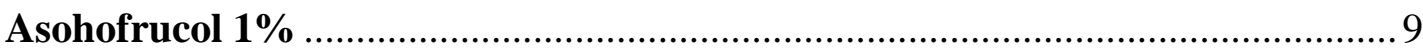

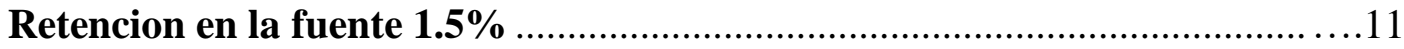

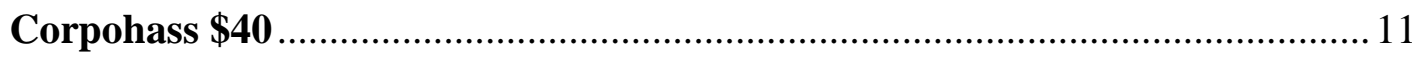

La Factura de Venta .......................................................................................13

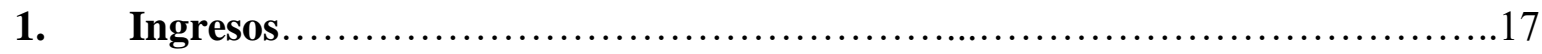

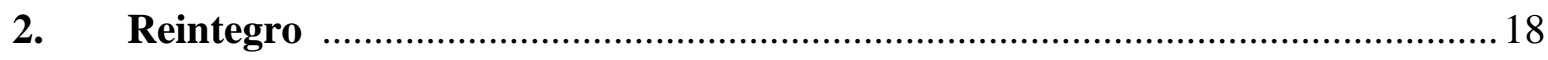

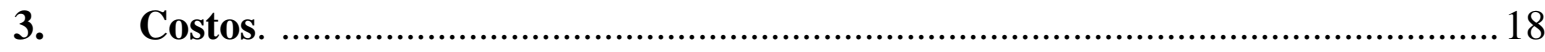

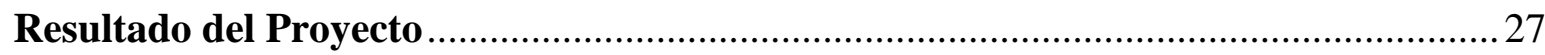

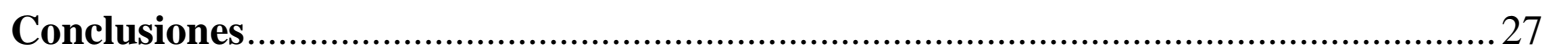

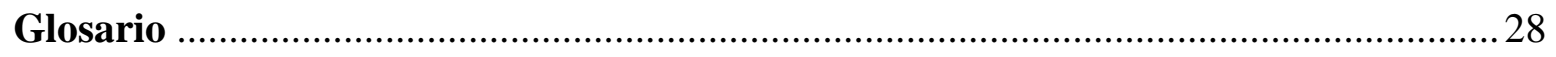

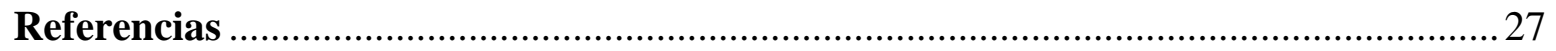




\section{Tabla de Ilustraciones}

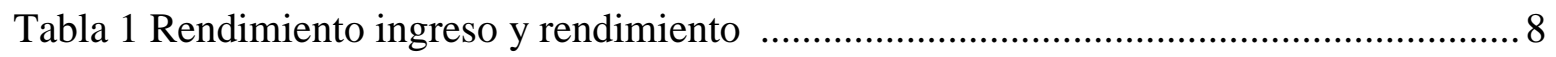

Tabla 2 Paso a paso de una exportación ........................................................................ 12

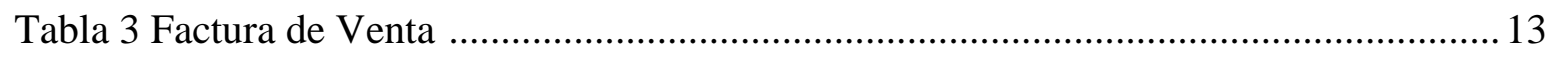

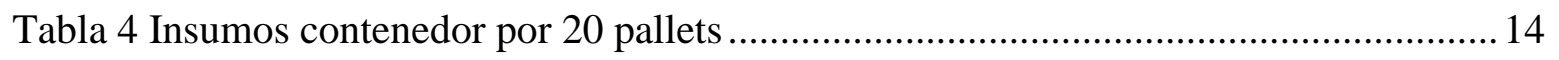

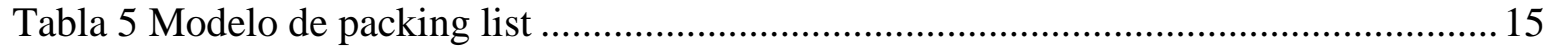

Tabla 6 Costos terceros para el envio de un contenedor ............................................. 16

Tabla 7 información necesaria de la factura de venta .................................................. 17

Tabla 8 Información de pagos según sus abonos …..................................................... 18

Tabla 9 Información necesari del packing list para hallar costo de la fruta .......................20

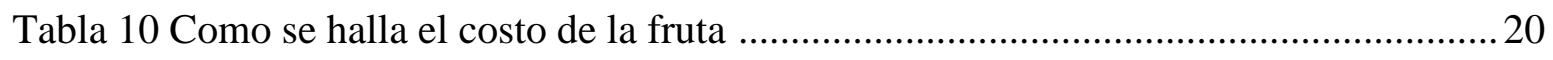

Tabla 11 Basados en costos po packing se halla la participación del predio en claibre.......21

Tabla 12 Basados en costos por packing se muestra participacion del pedio en $\mathrm{kg}$............21

Tabla 13 procedimeinto de reintegro menos los costos generados …..............................23

Tabla 14 Participación del predio en cajas enviadas ..................................................... 24

Tabla 15 Participación en dinero por cajas nviadas según su calibre ...............................25

Tabla 16 Participación d cada socio por predio................................................................26 


\section{Introducción}

Desde el momento en que una empresa se constituye, se debe tener claridad de la situación económica con que inicia, teniendo en cuanta los beneficios y los gastos que tendrá, lo cual se determinará aplicando técnicas que la contabilidad ofrece y parametriza. De igual manera se debe saber que al cabo del tiempo, la contabilidad sigue siendo muy necesaria para el buen funcionamiento, continuidad, estabilidad y trayectoria del negocio, proporcionando una información real y confiable de la situación financiera de la empresa, esto con el fin de poder tomar decisiones basados en la información que esta nos ofrece.

Es de suma importancia resaltar las ventajas que genera la contabilidad en una empresa, como por ejemplo saber con precisión que nos deben y que debemos, lo cual permite diferenciar los gastos que genera el mismo negocio, tener en cuenta los costos de producción, permitiendo de esta manera poner un valor-precio al trabajo elaborado, en caso de Tropy Fruits SAS la contabilidad nos permite determinar cuánto debemos cobrar al cliente por la maquila realizada y/o por fruta exportada entre muchas otras cosas. Es por eso que cuando la junta directiva de la empresa Tropy Fruits SAS deciden dar inicio a esta, utilizan las herramientas aportadas por la contabilidad, para tener información minuciosa del movimiento financiero que tiene la empresa y un presupuesto claro de cuantas exportaciones se tendrán que hacer mensualmente para pagar el sostenimiento de dicho negocio y lograr generar una utilidad.

Tropy fruits SAS cuenta con un contador público en planta junto con una auxiliar contable, con el fin de preparar y determinar de forma eficaz todos los estados financieros y contables, para no incurrir en sanciones o multas ante las entidades que supervisan el desarrollo de una empresa y que la junta directiva obtenga un acceso real y conciso de los resultados financieros que genera la actividad principal que desempeña la empresa a un plazo de tiempo definido. 
Resumen Ejecutivo

La empresa Tropy Fruits SAS es una empresa dedicada a la comercialización, maquila (proceso de selección por calibre y libre de cualquier enfermedad del aguacate hass, y el embalaje del mismo), y exportación de aguacate Hass. Aunque en principio los proveedores de fruta eran los mismos socios, hoy día debido a la demanda de aguacate hass, han tenido la necesidad de buscar más proveedores de fruta en el departamento de Antioquia y sus alrededores con el fin de poder cumplir y satisfacer la de manda y las necesidades de los clientes que se encuentran en países como: Estados Unidos, Perú, España, entre otros países de la Unión Europea.

En este informe se describirá el proceso e importancia que tiene la Auxiliar contable dentro de la compañía, describiendo cada una de sus funciones y el valor agregado que se le dará a la empresa dentro de este tiempo de desempeño 


\section{Breve Descripción de la Empresa}

"TROPY FRUITS SAS es una empresa exportadora de aguacate, conformado por 7 productores de aguacate hass los cuales se encuentran ubicados en: Urrao, Salgar, Santuario, Quindío, San Vicente, Angostura, Carmen de Atrato y Manizales, Cada uno de los predios están ubicados en diferentes pisos térmicos lo que proporciona un ciclo productivo de 10 meses aproximadamente al año.

La empresa tiene sus inicios en el año 2010 cuando los 7 productores colombianos (socios) se unieron para empezar con un proceso producción del aguacate hass. Después de 3 años, en el 2013 empezaron con la creación de la empresa Tropy Fruits SAS, empresa dedicada a la selección, empaque y exportación (proceso de maquila), materializando de esta manera el sueño de convertirse en productores exitosos, ofreciendo a los clientes el mejor aguacate hass, satisfaciendo los estándares internacionales más altos". (web: http://tropyfruits.com.co/.2013)

La empresa cuenta con una planta maquiladora por la variante a caldas en el municipio de La Estrella y está dividida en los siguientes departamentos:

- Gerente: Representante legal

- Departamento técnico: donde se encuentran los agrónomos Departamento de gestión humana: Encargada de la contratación de personal y afiliaciones respectivas

- Departamento de Contabilidad: Donde se encuentra el Contador público y la auxiliar contable (cargo que desempeño actualmente)

- Departamento de Producción; La cual esa en cabeza de la jefe de personal, seguido por el auxiliar de planta y los operarios. 


\section{Propuesta de Trabajo}

\section{Planteamiento del problema:}

La junta directiva de la empresa Tropy Fruits SAS dedicada a la exportación de aguacate hass, debe tener claro el costo que genera la exportación de un contendor de aguacate, es importante que cada uno de los datos proporcionados en estos costos estén bien sustentados y con su debido soporte.

Al iniciar, los socios eran los mismos proveedores, por lo cual es de suma importancia especificar cuánto gano o perdió en cada uno de los envíos; aunque la información se tiene contenida en los libros contables y en el sistema nunca se ha realizado un informe para la junta directiva donde se especifica cuanto se gana o se pierde por contenedor, y es a esto que se pretende llegar con este proyecto; cada vez que se despache un contenedor quede estipulado en un informe cuanta utilidad dejo.

\section{Justificación:}

Debido a algunas diferencias entre los socios se ve la necesidad de tener claro los costos por contenedor, puesto que entre ellos esta el mayor productor de aguacate hass de Antioquia y así mismo existe un socio el cual no es propietario de ningún predio; aquí la importancia de este informe, evitar nuevos diferencias entre ellos, también para poder tomar decisiones frente a las empresas con las que se trabaja a la hora del transporte del contendor o en casos de tramites de aduana ya que se hace por medio de terceros y minimizar dichos costos es también uno de los objetivos sin crear riesgos para la compañía.

Este informe lo intento gestionar la persona encargada anteriormente del puesto de auxiliar contable, realizando un cuadro en donde proporciono una información promediada, lo que deja muchos vacíos y muchos costos sin mencionar; por lo cual ahora se está realizando la información de una forma más detallada y con cada uno de sus soportes, otorgando de esta manera una información real a la junta directiva. 


\section{Desarrollo de la Práctica Profesional}

\section{Funciones:}

1. Recepción de facturación

2. Facturación

3. Cartera

4. Pago a proveedores

5. Conciliaciones Bancarias

6. Liquidación de nomina

7. Pago de nomina

8. Manejo de archivo

9. Manejo de software contable Agrowin
a. Creación de terceros
b. Reembolsos
c. Realización de comprobante de egreso
d. Realización de comprobante de ingreso
e. Realización de notas crédito
f. Realización de notas débito
g. Facturación,
h. Compras
i. Realización Órdenes de compra
j. Elaboración de requisiciones
k. Recepción de materiales
1. Generar certificados
$\mathrm{m}$. Manejo de inventarios

10. Configuración de permisos y perfiles para cada empelado del software, según su cargo

11. Realización de informes 


\section{Metodología de la Realización del Cuadro de Costos}

(Costos 2017 por factura)

Para llevar a cabo este proyecto se debe considerar y tener claridad en la transformación y lo que conlleva el proceso de exportación del aguacate, el cual se describirá a continuación:

1. Se hace el ingreso de la fruta por medio de la recepción de materiales, en este punto al llegar el aguacate a planta, se baja del camión el aguacate y se le da una identificación a la cual se le da el nombre de trazabilidad; este número de trazabilidad se determina por un número que se le da a cada predio (no se repite) y la fecha en la que está ingresando la fruta a la planta.

2. Luego que se determina esta trazabilidad se debe pesar el aguacate ingresado

3. Luego pasa por la maquiladora, en donde se hace una selección del aguacate por calibres $12,14,16,18,20,22,24,26,28-30$ o categoría II; la categoría II no sirve para exportación, se determina de este modo por tener algunas enfermedades que le da a el aguacate.

4. Después de seleccionado se pesa nuevamente para saber cuánto quedo para exportación, cuanto quedo en calibración y deshidratación, descarte (CAT II)

Con esta información se procede a realizar el Reporte de Ingreso y Rendimiento, el cual queda de la siguiente manera:

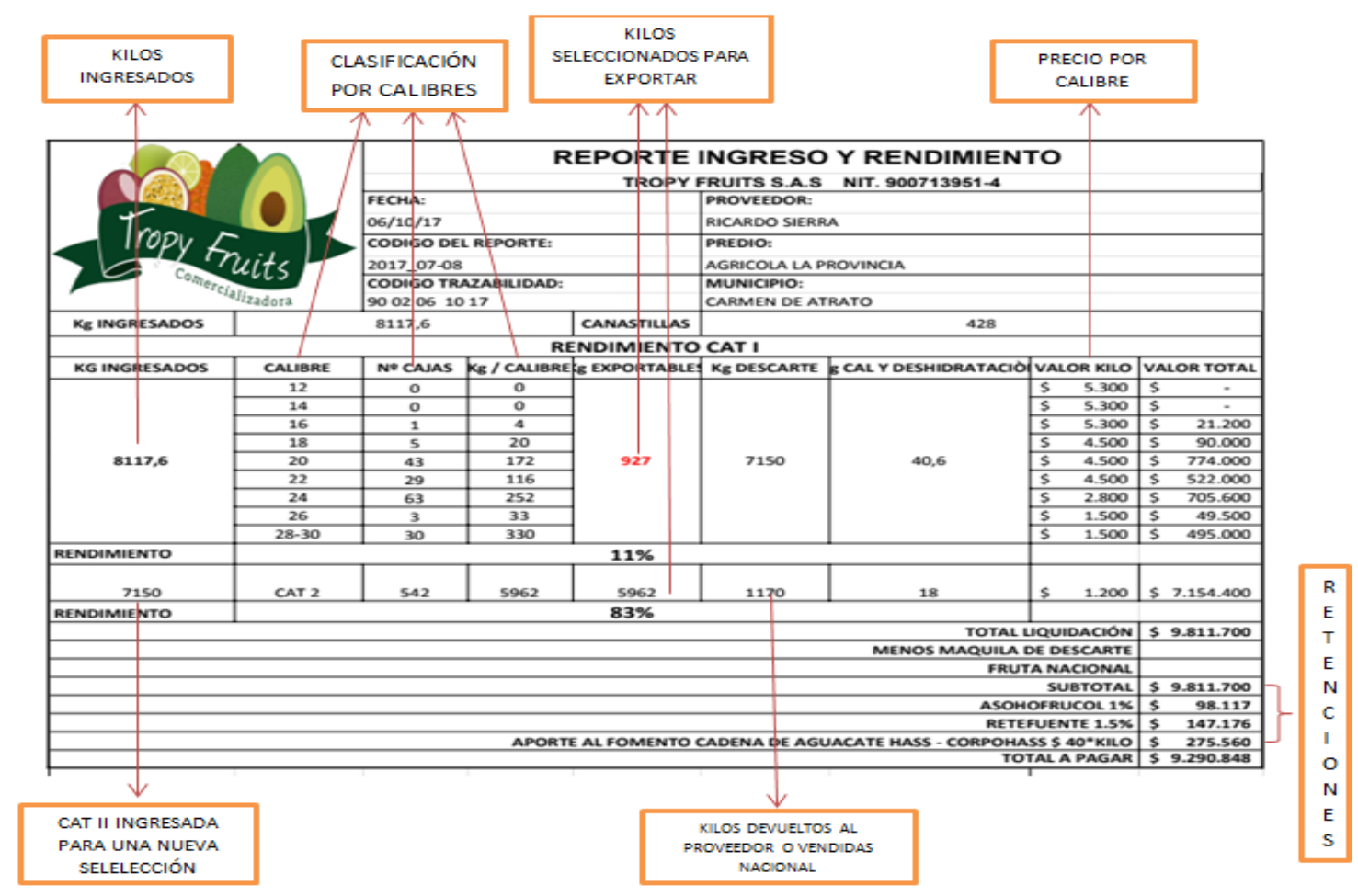




\section{Retenciones que se Realizan al Rendimiento}

\section{Asohofrucol 1\%}

"Es una cuenta especial de manejo, constituida por los recursos provenientes del recaudo de la Contribución Parafiscal Cuota de Fomento Hortofrutícola, creada mediante la Ley 118 de 1994.

Contribución Parafiscal cuota de fomento hortofrutícola: Es un gravamen obligatorio, de carácter parafiscal, el cual es utilizado en el desarrollo de los objetivos definidos por la Ley, en beneficio del subsector de las frutas y hortalizas.

\section{Objetivos del Fondo Nacional de Fomento Hortofrutícola.}

Los objetivos del Fondo Nacional de Fomento Hortofrutícola serán: Promover la investigación, prestar asistencia técnica, transferir tecnología, capacitar, acopiar y difundir información, estimular la formación de empresas comercializadoras, canales de acopio y distribución, apoyar las exportaciones y propender por la estabilización de precios de frutas y hortalizas, de manera que se consigan beneficios tanto para los productores como para los consumidores nacionales, y el desarrollo del Subsector. (Art. 15. Ley 118 de 1994.).

\section{¿Quiénes son los obligados a Pagar la Contribución Parafiscal Cuota de Fomento Hortofrutícola? \\ De conformidad con el Artículo 4 de la Ley 118 de 1994, modificado por el artículo 1 de la Ley 726 de 2001. Los productores de frutas y hortalizas ya sean personas naturales, jurídicas o sociedades de hecho, estarán obligados al pago de la Cuota de Fomento Hortofrutícola.}




\section{¿Cuándo se causa y se recauda la Contribución Parafiscal Cuota de Fomento Hortofrutícola, y cuál es su valor?}

La Cuota de Fomento Hortofrutícola, está constituida por el equivalente del uno por ciento (1\%) del valor de venta de frutas y hortalizas, (Artículo 3 de la Ley 118 de 1994.), y se causa en toda operación. Es decir, en cualquier nivel de la cadena de comercialización, procesamiento y transformación de las frutas y hortalizas, entiéndase éste, desde el productor hasta los posteriores agentes involucrados en la cadena.

El recaudador que acredite, mediante Paz y Salvo expedido por el administrador del fondo, la retención del pago de la cuota proveniente de la operación de venta que realicen los productores quedará exento de efectuar nuevamente el recaudo. Artículo 5 de la Ley 118 de 1994, modificado por el Artículo 2 de la Ley 726 de 2001.

\section{¿Quiénes son los responsables de efectuar el recaudo de la Contribución Parafiscal} Cuota de Fomento Hortofrutícola?

El Artículo 5 de la Ley 118 de 1994, modificado por el artículo 2 de la Ley 726 de 2001, define que, serán recaudadores de la Cuota de Fomento Hortofrutícola, las personas naturales o jurídicas y las sociedades de hecho, que procesen o comercialicen frutas u hortalizas, conforme a la reglamentación que para el efecto expida el Gobierno Nacional. Dicho artículo fue reglamentado en los artículos 1 (Ámbito de Aplicación), y 2 (Definiciones) del Decreto 3748 de 2004". (REF. http://www.asohofrucol.com.co/interna.php?cat=4\&scat=27\&act=1) 


\section{Retención en la Fuente $1.5 \%$}

"Retención conocida como Compra de productos agrícolas o pecuarios y sus tarifas de retención en la fuente y la cual Conforme a lo señalado en el artículo 1 del Decreto 2595 de 1993, a los pagos o abonos en cuentas que se originen por la adquisición de productos agrícolas o pecuarios sin procesamiento industrial se les aplicará una tarifa de retención en la fuente a título de renta del $1,5 \%$. No obstante, dicha retención se efectuará sobre pagos o abonos en cuenta superiores a 92 UVT, lo anterior debido a que con el numeral 82 del artículo 868-1 del ET se expresó en UVT el valor de \$1.759.000 indicado en el artículo en mención” . .REF. http://actualicese.com/2016/10/04/compra-de-productos-agricolas-opecuarios-y-sus-tarifas-de-retencion-en-la-fuente/)

\section{Corpohass $\$ 40$ por kilo Exportable}

"El objeto de la Corporación es orientar, fomentar y proyectar la producción y comercialización de Aguacate Hass en Colombia, procurando el bienestar del productor y del exportador.

\section{Beneficios de ser asociado de Corpohass}

- Representatividad Gremial, que haga que se consolide el desarrollo de la industria.

- Elegir o ser elegido para los cargos de la Corporación.

- Buscar articular el eje productor-gremio gobierno, para tener acceso a nuevos mercados

- Participación en gestión de proyectos de interés colectivo.

$\checkmark$ Proyectos de Certificaciones ajustadas a la industria.

$\checkmark$ Capacitaciones en todos los eslabones de la cadena.

$\checkmark$ Consecución de líneas crediticias favorables.

$\checkmark$ Consolidar una cultura exportadora en las regiones.

$\checkmark$ Promoción al consumo de Aguacate Hass en el mercado interno.

- Acceso a insumos con precios más asequibles dada la posibilidad de compras por volumen e importación directa.

- Acceso permanente a información de carácter técnico, comercial y gremial.

Se financia cobrando $\$ 40$ por kilo de la fruta que se va a exportar". (REF. Presentación powerPoint/ CORPOHASS - El Gremiod de la Agroindustria de Aguacates Hass). 


\section{Universidad Santo Tomas PRIMER CLAUSTRO UNIVERSITARIO DE COLOMBIA

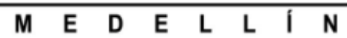

Después de ingresada la fruta y seleccionada (maquila), se procede con el ingreso de la información en el sistema que se maneja en la empresa, el cual se llama AGROWIN y así mismo proceder al pago de cada proveedor basados en el reporte de ingreso y rendimiento; de la manera como se hace físicamente también se hace en el sistema, de la siguiente manera:

\begin{tabular}{|c|c|c|}
\hline \multicolumn{3}{|c|}{ INGRESO DE FRUTA A PLANTA } \\
\hline PASO No. & FISICAMENTE PLANTA & EN EL SISTEMA AGOWIN \\
\hline 1 & $\begin{array}{l}\text { Ingresa la fruta en kilos y en todos los } \\
\text { calibres solo de un predio a la vez (se } \\
\text { recibe proveedor por proveedor) }\end{array}$ & $\begin{array}{l}\text { En el sistema ingresa en una Recepción de } \\
\text { materiales por el total de los kilos (sin } \\
\text { especificar calibres) a nombre del predio del } \\
\text { proveedor el cual será la bodega }\end{array}$ \\
\hline 2 & Se maquila y se selecciona por calibres & $\begin{array}{l}\text { Se realiza la factura de compra en base al } \\
\text { reporte de ingreso y rendimiento bajo la } \\
\text { bodega (nombre del predio) }\end{array}$ \\
\hline 3 & $\begin{array}{l}\text { se paga a cada proveedor basados en la } \\
\text { maquila realizada de fruta, ya que cada } \\
\text { calibre tiene un precio }\end{array}$ & $\begin{array}{l}\text { se procede hace el comprobante de egreso } \\
\text { para proceder al pago basados en el reporte } \\
\text { de ingreso y rendimiento }\end{array}$ \\
\hline 4 & $\begin{array}{l}\text { se ingresa a cava, que es el cuarto frio } \\
\text { en el cual deben permanecer mínimo } \\
24 \text { horas antes de la exportación, aquí } \\
\text { se van llevando de todos los predios } \\
\text { luego de cada maquila. Se puede llegar } \\
\text { a tener fruta en una sola cava de } 5 \\
\text { proveedores }\end{array}$ & $\begin{array}{l}\text { En el sistema se realiza un Orden de } \\
\text { producción en el cual se transforma de cada } \\
\text { bodega por predio a una bodega general en } \\
\text { donde habrá todos los calibres }\end{array}$ \\
\hline 5 & $\begin{array}{l}\text { Después del proceso de Frio, se } \\
\text { procede a armar los pallets que por lo } \\
\text { general son } 20 \text { por contenedor, en } \\
\text { donde hay cajas de } 4 \mathrm{~kg} \text { y de } 11 \mathrm{~kg}, \\
\text { marcados con sus respetivos predios, } \\
\text { calibres y peso en kg }\end{array}$ & $\begin{array}{c}\text { En el sistema se hace una transformación por } \\
\text { el envió de kg de cada predio que se saca de } \\
\text { la cava, basados en el packing list }\end{array}$ \\
\hline 6 & $\begin{array}{r}\text { Se Finaliza realizando la factura de venta } \\
\text { se entre }\end{array}$ & $\begin{array}{l}\text { del contenedor cargado y que posteriormente } \\
\text { gara al cliente }\end{array}$ \\
\hline 7 & & $\begin{array}{c}\text { Cuando el cliente pague, se realiza en agrowin } \\
\text { un recibo de ingreso cancelando el valor de la } \\
\text { factura }\end{array}$ \\
\hline
\end{tabular}

Ilustración 2 Paso a Paso de una exportación 


\section{La factura de venta:}

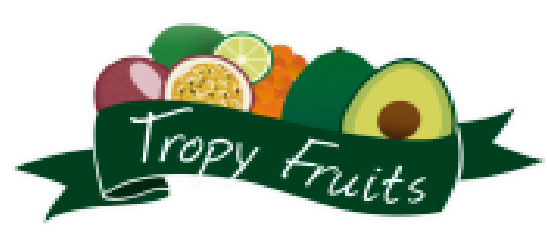

SELL.ER

TROPYFRUITS SAS

CRA $47 \approx 7.41$

MEDELLLIN - COL.OMBIA

0057-3128660315

perencinetrogoffruitscom

NIT 900.713 .951 .4
CUSTOMER

№ 222

GEORCE HELFER SA

1 RUE DE TROPIQUES ENT 133

94538 RUINGIS- FRANCE

$0033 \cdot 1 \cdot 45123650$

isabellepieraertsghelfertrance.fr

RESOLUCION DLAN:RESOLUCION N' 110000649975 FECHA 201510109 AUTORIZADA DESDE N'57 HASTA EL N* 1000

SALESWOMAN

GLoRIA SANIN

TERMS

B DAYS CREDIT
DATE

enero 2 DE 2017

INCOTERM

FDB/SANTA MeARTA
ORDER

LINE

MAERSK

VESSEL. / VOYAGE

HAM MONIA EMDEN 1702
CONTAINER

CURRENCY

EURO
QTY BOXES TTEM

2.376

792

792

264

192

192
AGUACATE HASS 4KG CALIBRE 14 * 18

ACUACATE HASS 4KG CALIBRE 20

AGUACATE HASS 4KG CALIBRE 27

AGUACATE HASS 4 KG CAL.IBRE 24

AGUACATE HASS 11 KG CALIBREE 26

AGUACATE HASS 11KG CAL.IBRE 28.30
UNIT VALUE

$\epsilon 9,00$
$\epsilon 8,00$
$\epsilon 7,00$
$€ 5,00$
$€ 10,00$
$€ 8,50$

TOTAL

ez1.384,00

e6.336,00

$\epsilon 5.544,00$

$€ 1.320,00$

$€ 1.920,00$

$\in 1,632,00$

BANK: BANCOLOMBIA

BENEFICIARY: TROPYFRUITS SAS

ACCDUNT: 34222483265

SWFIT CODE: COLOCOEM

VALUE:

\begin{tabular}{lr}
\hline Subtotal & $e 38.136,00$ \\
& \\
Taxes & $e 0,00$ \\
Others & $e 0,00$ \\
Total & $\epsilon 38.136,00$ \\
\hline
\end{tabular}

TERMS OF THE SALE

DESCRIPTION

ADVANCE PAYMENT 60\% ACAINST SHIPPING

DOCUMENTS AND PENDENG AMEOUNT

40\% 8 DAYS AFTER ARRIVAL.

$\begin{array}{lc}\text { 20 PALIETS } & 4608 \text { BOXES } \\ \text { NET WEIGHT } & 21120 \mathrm{KC} \\ \text { GROSS WEIGHT } & 22300 \mathrm{KG}\end{array}$

TROPYFRUITS SAS

GEORCE HELFER SA

IMPORTENTE: ESTA FACTURA DE VENTA SE ASIMILA PARA TODOS SUS EFECTOS A LA LETRA DE CAMBIO 774 DEL. C DE C EL CLIENTE ACEPTA QUE LAS PERSONAS QUE FIRMLAN LA PRESENTE TIENEN AUTORIZACION PARA ELL.O Y POR LO TANTO SON ESE ACTO REPRESENTANTES DEL. CLIENTE EL. CUAL SE HACE RESPONSABL.F DE LA CANCELACION, LA MERCANCIA HA SIDO RECIBIDA REAL Y MUATERIALMENTE A SATISFACOON EN CASO DE MORA SE COBRARAN INTERESES LA TASA MAXIMEA PERMTTIDA POR LA LEY

COMERCIALIZADORA DE PRODUCTOS AGRUCOLAS

NO SOMOS AUTORETENEDORES - NOS SOMOS GRANDES CONTHBBUYNTES

Ilustración 3 factura de venta 
Como se expresa en la tabla ilustración 2 anteriormente, después de tener la cantidad requerida para exportar (según negociación con el cliente) se procede a armar los pallets, los cuales irán al contendedor que por lo general en una exportación de 21.120 kilos de aguacate son 20 pallet. Para cada pallet se necesitan unos insumos como lo son:

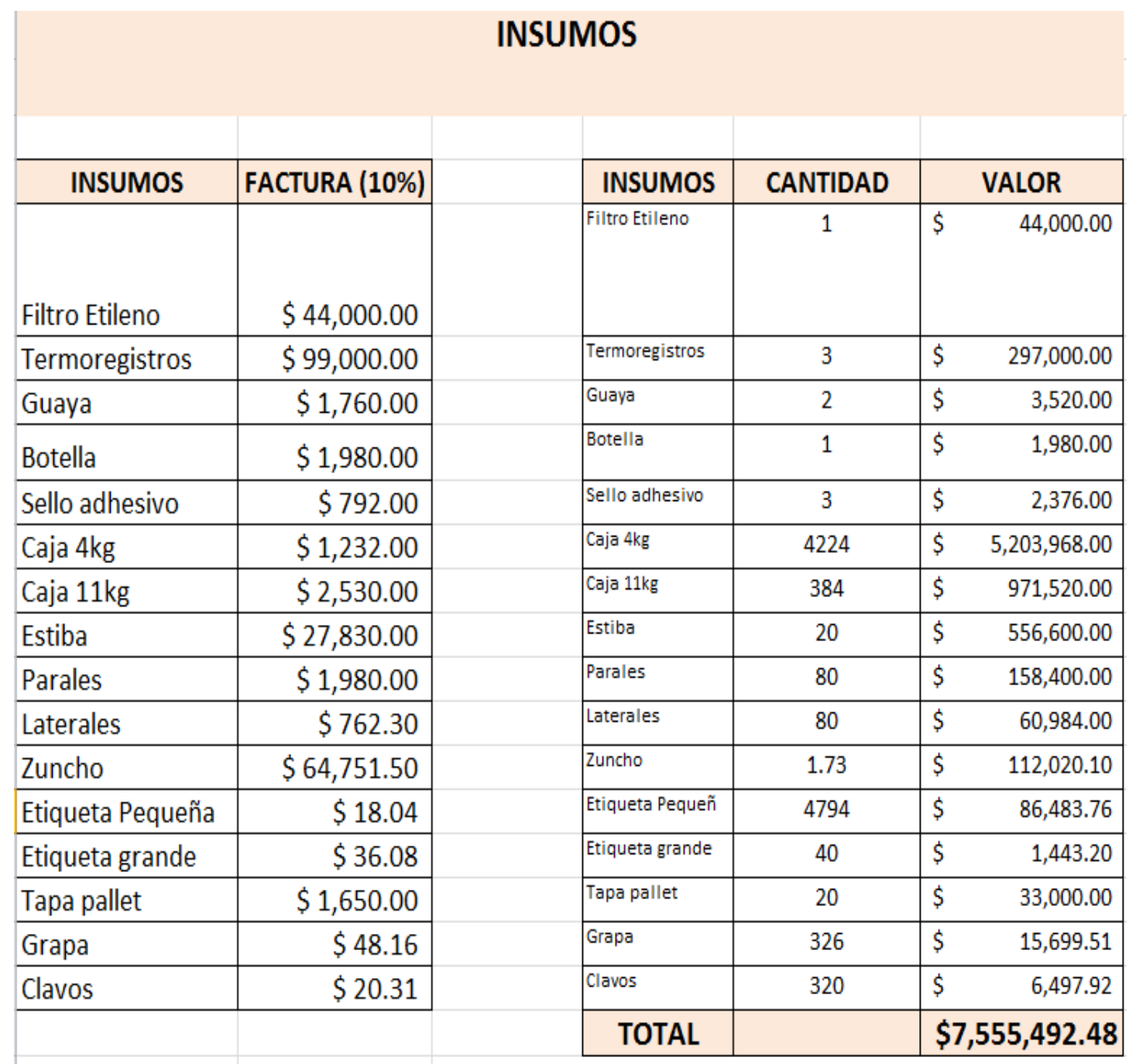

Ilustración 4 Insumos por 1 contenedor de 20 pallets

De igual manera cando se va a enviar un contendor se debe saber la participación de cada proveedor en dicha negociación, la cual queda registrada en un packing list, este es el medio por el cual se guarda la información de la fruta que se exportara, relacionando en él el predio, 
cantidad envida y calibre; esta fruta sale en cajas que según el calibre puede ser de 4 kilos o de 11 kilos. En un pallet que contienen cajas de 4 kilos en total caben 264 cajas, cuando el Pallet contiene cajas de 11 kilos caben 102 cajas. Y la presentación del packing list será la siguiente:

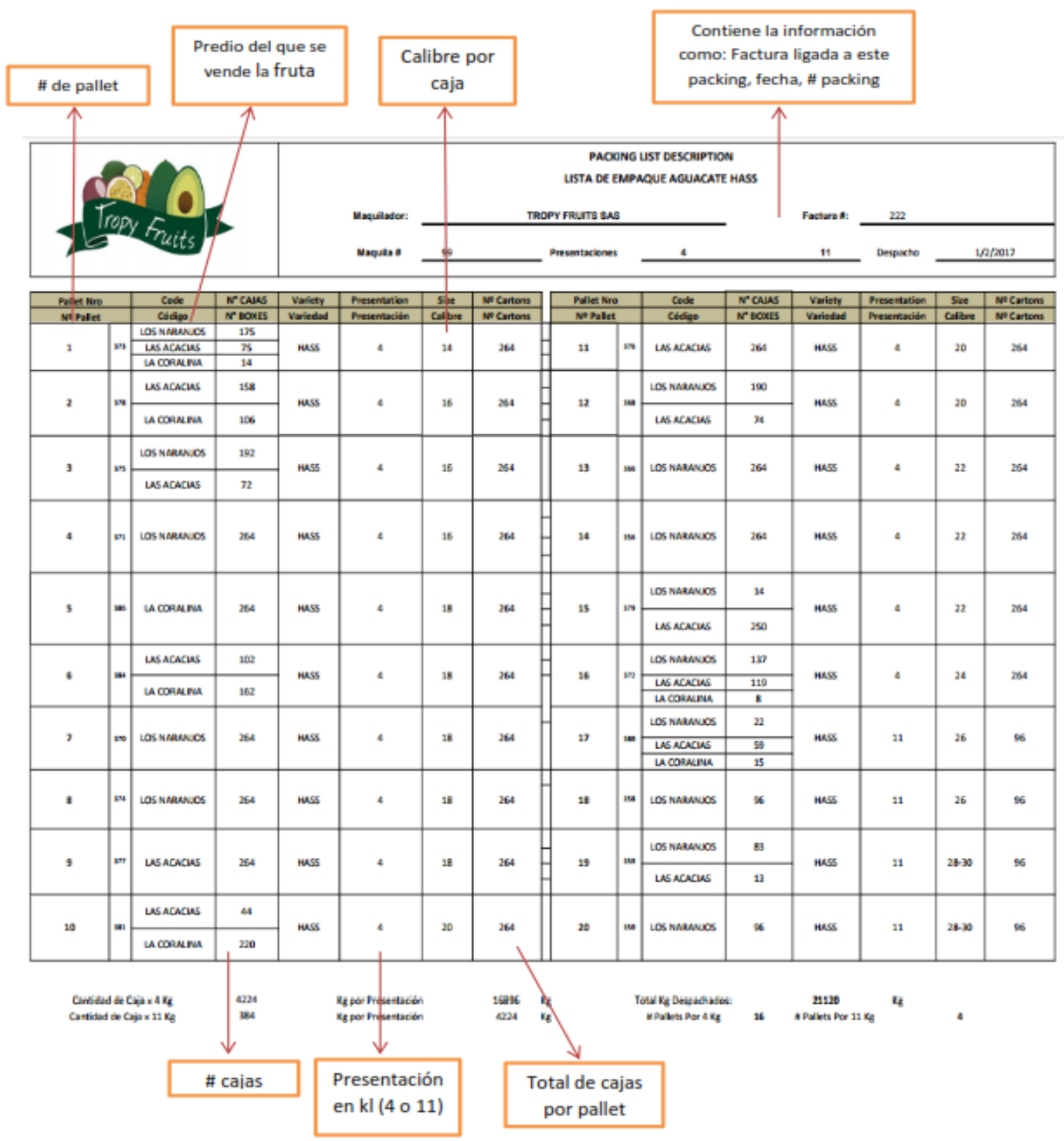

Ilustración 5 Modelo de Packing List 
Después de que el contenedor quede con la carga lista, se procede a llevarlo al puerto que puede ser el de Cartagena, santa marta o turbo, que son con los que se trabaja actualmente. Esto requiere de otros gastos como lo son:

- Transporte desde la planta hasta el puerto: Se ha trabajado con empresas como planet cargo, ega kat, operadora portuaria del caribe, olt transportes

- Conexión de energía hasta el embarque del contenedor: ya que por ser fruta requiere de refrigeración: Este servicio lo presta la empresa Compañía Frutera de Sevilla (banacol)

- Los gastos de aduana: Este servicio lo prestaba planet cargo, pero por costos elevados en la actualidad se trabaja con Banaduana.

En la actualidad los costos por transporte de contenedor hasta el puerto de embarque son los siguientes:

\section{TERCEROS}

\begin{tabular}{|c|c|c|}
\hline TERCERO & VALOR & DESCRIPCIÓN \\
\hline BANADUANA & $\$ 1,073,688.00$ & $\begin{array}{l}\text { ADUANA, ALQU. DE CONTENEDOR, COURRIER, } \\
\text { GTOS DEPUERTO,SEGURO,MANEO,IVA } \\
\text { GENERADO }\end{array}$ \\
\hline OPERADORA PORTUARIA & $\$ 3,316,500.00$ & TRANSPORTE+COMBUSTBBLE \\
\hline CIA FRUTERA DE SERVICIOS & $\$ 1,709,957.00$ & CONEXIÓN DEENERGIA \\
\hline TOTAL & $\$ 6,100,145.00$ & \\
\hline
\end{tabular}

Ilustración 6 Costos de terceros para el envió de un contenedor 
Después del procedimiento anterior y del reintegro de divisas (pago del cliente a Tropy Fruits), es necesario saber qué utilidad deja la exportación del contenedor a cada uno de los socios y el costo real en los que se debe incurrir, es aquí donde empieza el proyecto a presentar, es un archivo en Excel donde se muestra el ingreso (pago de los clientes), menos cada uno de los costos y posteriormente la repartición de utilidad al socio según corresponda (teniendo en cuenta que no todos los proveedores de fruta son socios).

El cuadro lleva por nombre "Costos" y se divide en 4 grandes partes:

1. Ingresos: En esta primera parte del cuadro, lo encontramos subdividido en tres partes, la primera sección: la facturación (cada factura es un envió del contenedor), donde se describe la fecha de la factura, su respectivo número, el número de packing list al que corresponde, el nombre del cliente, y el valor en la moneda que se hizo el negocio (Peso, Dólar, Euro).

\begin{tabular}{|c|c|c|c|}
\hline \multicolumn{3}{|c|}{ FACTURACION } \\
\hline FECHA & FACTURA & PACKING & VR POR \\
LIST & FACTURA \\
\hline 22 -jun-17 & 260 & 125 & $£ 40.491,00$ \\
\hline 22 -jun-17 & 261 & 126 & $£ 47.124,00$ \\
\hline 22 -jun-17 & 262 & 127 & $£ 30.855,00$ \\
\hline 29 -jun-17 & 263 & 128 & $£ 26.746,50$ \\
\hline 7 -jul-17 & 267 & 129 & $£ 39.960,00$ \\
\hline 4 -ago-17 & 269 & 130 & $£ 41.043,75$ \\
\hline 11 -ago-17 & 275 & 131 & $£ 38.681,25$ \\
\hline
\end{tabular}

Ilustración 7 información necesaria de cada Factura de Venta 
2. Reintegro - Ajuste: En esta sección se encuentra los abonos que hace el cliente a la factura (en la moneda que se negocia), Notas Debito, Notas crédito según se hallan tenido que realizar (bien sea por que la fruta se dañó en el transcurso del viaje hasta la entrega o porque el cliente la vendió a mayor valor y lo reconoce a la empresa), estas casillas se suman y tiene que dar el valor igual al de la factura, también se encuentra una casilla donde se expresa a cuanto corresponde ese valor en pesos (valor recibido en pesos colombianos).

\begin{tabular}{|c|c|c|c|c|c|c|c|}
\hline \multicolumn{5}{|c|}{ REINTEGRO } & & \multicolumn{2}{|c|}{ AJUSTE } \\
\hline $\begin{array}{l}\text { REINTEGRO } \\
\text { (EUROS) }\end{array}$ & & $\begin{array}{l}\text { TAL REINTEGRO } \\
\text { (PESOS) }\end{array}$ & ABONO 1 & ABONO 2 & ABONO 3 & ND & NC \\
\hline$£ 24.235,10$ & & $79.871 .464,00$ & $£ 12.117,55$ & $£ 12.117,55$ & & & \\
\hline$£ 40.309,50$ & & $138.203 .395,00$ & $£ 28.216,65$ & $£ 12.092,85$ & & & \\
\hline$£ 40.491,00$ & & $138.825 .678,00$ & $£ 28.343,70$ & $£ 12.147,30$ & & & \\
\hline$£ 47.124,00$ & $\$$ & $161.567 .292,00$ & $€ 32.986,80$ & $£ 14.137,20$ & & & \\
\hline$€ 30.855,00$ & $\$$ & $105.788 .108,00$ & $£ 21.598,50$ & $€ 9.256,50$ & & & \\
\hline$£ 26.746,50$ & $\$$ & $91.608 .689,00$ & $£ 13.373,25$ & $13.373,25$ & & & \\
\hline$£ 27.972,00$ & $\$$ & $96.953 .207,00$ & $£ 27.972,00$ & & & & \\
\hline$£ 28.724,63$ & $\$$ & $98.458 .598,00$ & $£ 28.724,63$ & & & & \\
\hline$£ 19.334,63$ & $\$$ & $67.191 .538,00$ & $£ 19.334,63$ & & & & \\
\hline
\end{tabular}

Ilustración 8 Información del Pago según abonos

\section{COSTOS}

En esta tercera parte encontraremos todos los costos que sobrelleva la exportación de cada contenedor, como lo es a:

- Una administración que corresponde al $4 \%$ de la utilidad de este (manejo que hace Tropy fruits de la operación de exportación)

- El costo de la fruta el cual se calcula de cada packing list, teniendo en cuenta el predio, lo que se le pago, el calibre que exporto cada predio y la cantidad de kilos. 
Ejemplo:
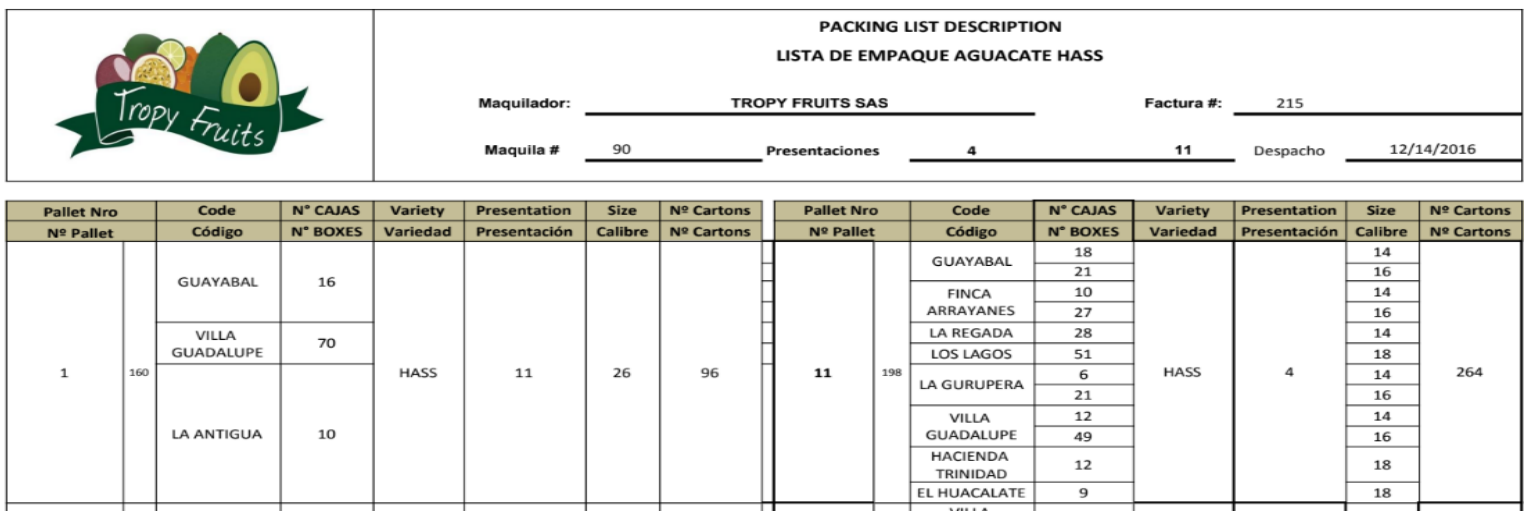

Ilustración 9 Información necesaria del packing para hallar el costo de la fruta enviada

En el primer pallet, tenemos que la fruta se envió distribuida de la siguiente manera:

a. El predio Guayabal envió 16 cajas calibre 26 en una caja de 11 kilos, suponiendo que al proveedor del predio guayabal se le pago el calibre 26 a $\$ 4.000$ (este valor real se obtiene de la factura de compra y/o rendimiento) ; teniendo esto en cuenta se procede a hallar el costo de este ítem, y para ello hacemos la siguiente operación:

16 cajas $\boldsymbol{x} 11 \boldsymbol{x} \$ 4.000=\$ 704.000$

Este sería el costo real de este ítem, así se hace uno por uno y finalmente se suma cada ítem, lo que da como resultado es el costo total de la fruta por packing list. 
\begin{tabular}{lllllllll}
$M$ & E & D & E & L & L & I & N \\
\hline
\end{tabular}

\begin{tabular}{|c|c|c|c|c|c|c|c|c|c|}
\hline \multicolumn{10}{|c|}{ COSTO POR PACKING LIST } \\
\hline PALET & CALIBRE & PREDIO & PRESENTACIÓN (KG) & N. DECAJAS & & RCALIBRE & & TOTAL & KILOS \\
\hline 1 & 14 & LACORALINA & 4 & 5 & $\$$ & $5.200,00$ & 5 & $104.000,00$ & 20 \\
\hline 1 & 14 & LASACACIAS & 4 & 39 & 5 & $5.200,00$ & $\$$ & $811.200,00$ & 156 \\
\hline 1 & 16 & LACORALINA & 4 & 66 & 5 & $4.900,00$ & $\$$ & $1.293 .600,00$ & 264 \\
\hline 1 & 16 & LASACACIAS & 4 & 143 & $\$$ & $4.900,00$ & $\$$ & $2.802 .800,00$ & 572 \\
\hline 1 & 18 & LASACACIAS & 4 & 11 & $\$$ & $4.900,00$ & $\$$ & $215.600,00$ & 44 \\
\hline 2 & 18 & LACORALINA & 4 & 55 & $\$$ & $4.900,00$ & $\$$ & $1.078 .000,00$ & 220 \\
\hline 2 & 18 & LASACACIAS & 4 & 209 & $\$$ & $4.900,00$ & 5 & $4.096 .400,00$ & 836 \\
\hline $3 A 6$ & 20 & LACORALINA & 4 & 362 & $\$$ & $4.800,00$ & 5 & $6.950 .400,00$ & 1448 \\
\hline $3 A 6$ & 20 & LASACACIAS & 4 & 646 & $\$$ & $4.800,00$ & $\$$ & $12.403 .200,00$ & 2584 \\
\hline $6 A 8$ & 22 & LASACACIAS & 4 & 347 & $\$$ & $4.500,00$ & 5 & $6.246 .000,00$ & 1388 \\
\hline $6 A 8$ & 22 & LACORALINA & 4 & 301 & 5 & $4.500,00$ & 5 & $5.418 .000,00$ & 1204 \\
\hline $6 A 8$ & 22 & LAMONTERA & 4 & 63 & $\$$ & $4.500,00$ & $\$$ & $1.134 .000,00$ & 252 \\
\hline $9 A 12$ & 24 & LAMONTERA & 4 & 94 & $\$$ & $4.000,00$ & 5 & $1.504 .000,00$ & 376 \\
\hline $9 A 12$ & 24 & LACORALINA & 4 & 297 & $\$$ & $4.000,00$ & $\$$ & $4.752 .000,00$ & 1188 \\
\hline $9 A 12$ & 24 & LASACACIAS & 4 & 530 & $\$$ & $4.000,00$ & $\$$ & $8.480 .000,00$ & 2120 \\
\hline $13 \mathrm{~A} 20$ & $28-30$ & VERACRUZ & 11 & 214 & $\$$ & $1.500,00$ & $\$$ & $3.531 .000,00$ & 2354 \\
\hline $13 \mathrm{~A} 20$ & $28 \cdot 30$ & FINCA BESARABIA & 11 & 32 & 5 & $1.000,00$ & $\$$ & $352.000,00$ & 352 \\
\hline $13 \mathrm{~A} 20$ & $28 \cdot 30$ & SAN JOSE & 11 & 61 & $\$$ & $1.000,00$ & $\$$ & $671.000,00$ & 671 \\
\hline $13 \mathrm{~A} 20$ & $28-30$ & LACORALINA & 11 & 257 & $\$$ & $1.500,00$ & 5 & $4.240 .500,00$ & 2827 \\
\hline $13 \mathrm{~A} 20$ & $28 \cdot 30$ & LAMONTERA & 11 & 47 & $\$$ & $1.500,00$ & $\$$ & $775.500,00$ & 517 \\
\hline $13 \mathrm{~A} 20$ & $28 \cdot 30$ & LASACACIAS & 11 & 205 & $\$$ & $1.500,00$ & $\$$ & $3.382 .500,00$ & 2255 \\
\hline & & TOTAL & & 3984 & & & $\$$ & $70.241 .700,00$ & 21648 \\
\hline
\end{tabular}

Ilustración 10 Como hallar el costo de la futa

Este dato se tiene en una hoja adicional como soporte de todo el proceso factura por factura; en esta hoja que se denomina "costo fruta por packing" se adicionan dos graficas una donde se expresa la participación del predio por calibre, y la otra donde se muestra la participación del predio por participación en kilos. Todo esto para mostrar una información más clara a la junta directiva. 


\begin{tabular}{|c|c|r|}
\hline \multicolumn{3}{|c|}{ PARTICIPACIÓN POR KILOS } \\
\hline CALIBRE & PREDIO & \multicolumn{1}{c|}{ PORCENTAJE } \\
\hline 14 & LACORALINA & $0,09 \%$ \\
\hline 14 & LASACACIAS & $0,72 \%$ \\
\hline 16 & LACORALINA & $1,22 \%$ \\
\hline 16 & LAS ACACIAS & $2,64 \%$ \\
\hline 18 & LASACACIAS & $0,20 \%$ \\
\hline 18 & LACORALINA & $1,02 \%$ \\
\hline 18 & LAS ACACIAS & $3,86 \%$ \\
\hline 20 & LACORALINA & $6,69 \%$ \\
\hline 20 & LASACACIAS & $11,94 \%$ \\
\hline 22 & LAS ACACIAS & $6,41 \%$ \\
\hline 22 & LACORALINA & $5,56 \%$ \\
\hline 22 & LAMONTERA & $1,16 \%$ \\
\hline 24 & LAMONTERA & $1,74 \%$ \\
\hline 24 & LACORALINA & $5,49 \%$ \\
\hline 24 & LASACACIAS & $9,79 \%$ \\
\hline $28-30$ & VERACRUZ & $10,87 \%$ \\
\hline $28-30$ & FINCABESARABIA & $1,63 \%$ \\
\hline $28-30$ & SAN JOSE & $3,10 \%$ \\
\hline $28-30$ & LACORALINA & $13,06 \%$ \\
\hline $28-30$ & LAMONTERA & $2,39 \%$ \\
\hline $28-30$ & LAS ACACIAS & $10,42 \%$ \\
\hline
\end{tabular}

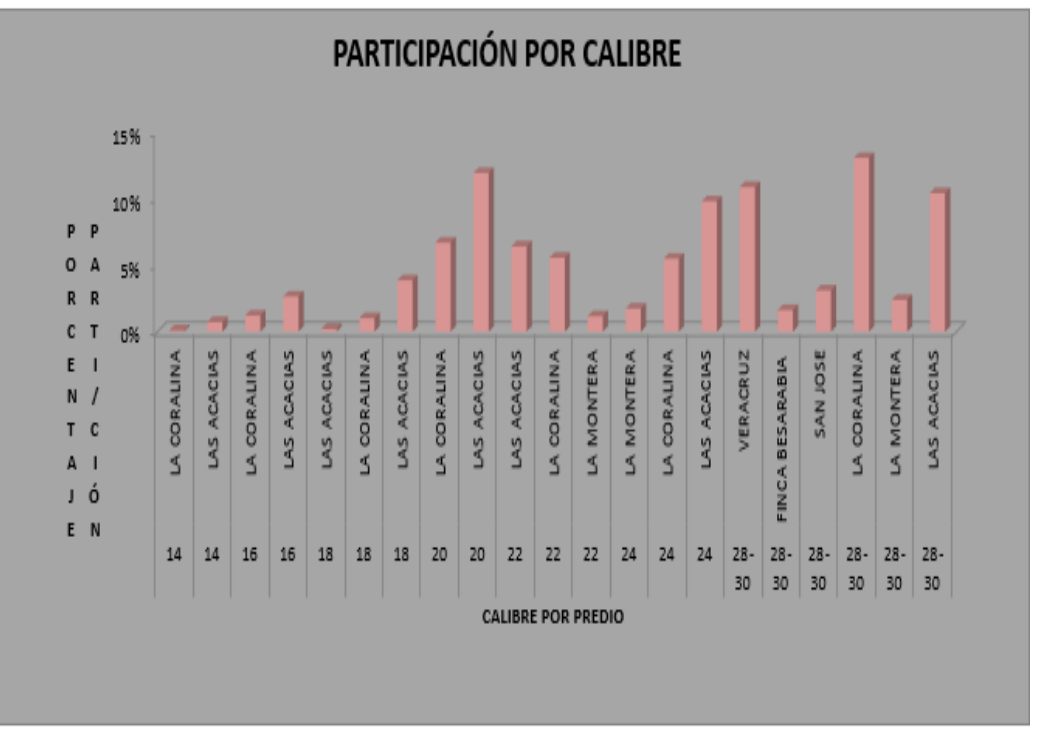

Ilustración 11 Basados en el costo por packing se halla participación de cada predio en calibre

\begin{tabular}{|c|c|c|c|c|c|}
\hline \multicolumn{6}{|c|}{ PARTICIPACIÓN } \\
\hline PREDIO & KILOS & $\% \mathrm{KG}$ & & OTAL & \% TOTAL \\
\hline LA CORALINA & 7171 & 33,1396 & 5 & $23.836 .500,00$ & $33,93 \%$ \\
\hline LAS ACACIAS & 9955 & 45,9996 & 5 & $38.437 .700,00$ & $54,72 \%$ \\
\hline LA MONTERA & 1145 & 5,2996 & 5 & $3.413 .500,00$ & 4,8696 \\
\hline VERACRUZ & 2354 & 10,8796 & $\$$ & $3.531 .000,00$ & 5,0396 \\
\hline FINCA BESARABIA & 352 & 1,6396 & $\underline{s}$ & $352.000,00$ & 0,5096 \\
\hline SAN JOSE & 671 & 3,1096 & 5 & $671.000,00$ & 0,9696 \\
\hline TOTAL & 21648 & $100,00 \%$ & $\$$ & $70.241 .700,00$ & $100,00 \%$ \\
\hline
\end{tabular}

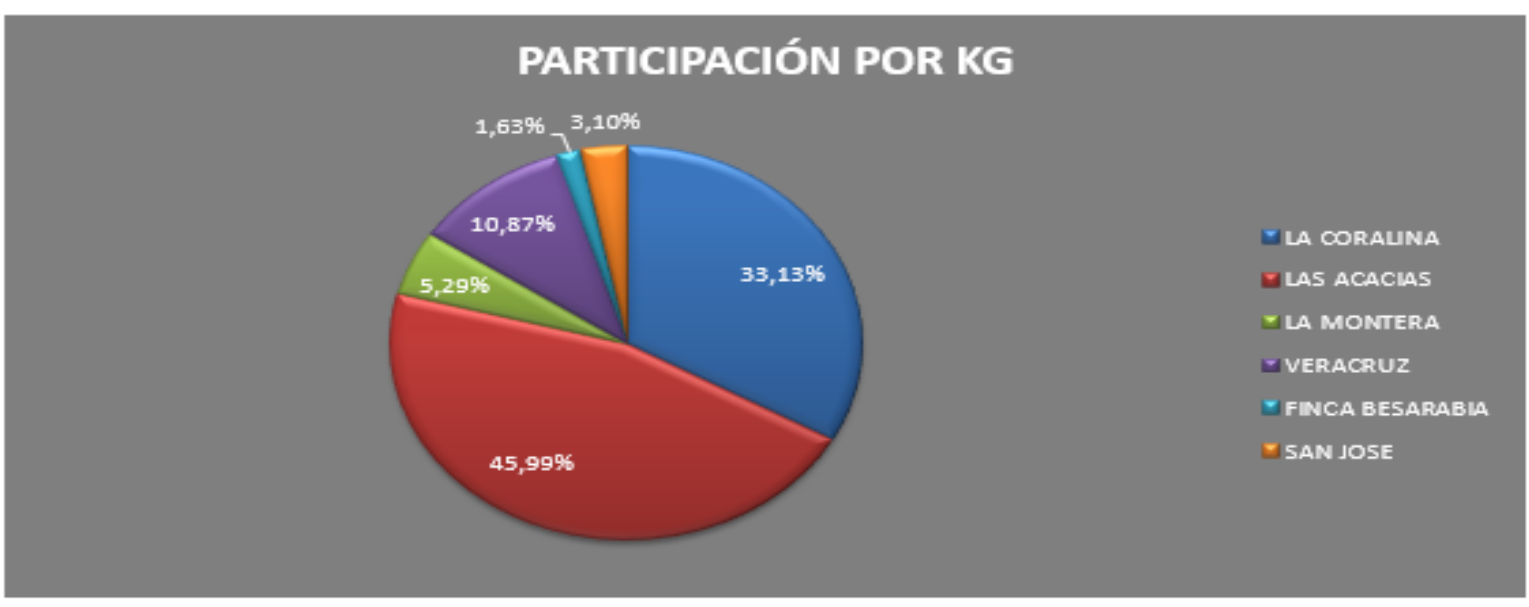

Ilustración 12 Basados en el costo por paking se muestra la participación por predio en kg 
- Otra casilla de esta tercera parte se llama "kilos" que corresponde a los kilos que se exportaron en esa factura. exportado y va unido a "maquila", esta comprende lo relacionado a los CIF, Mano de obra, materia prima etc., se tomó una base promedio lo que corresponde a que por 21.120 kilos exportados genera un costo de CIF, mano de obra, materia prima, etc., de $\$ 13.500 .000$, en caso de que se exporte más cantidad de kilos se realiza una regla de tres para hallar el valor del costo de la maquila, si los kilos exportados es menor la base de la maquila seguirá siendo de $\$ 13.500 .000$.

Ejemplo A: se exportan 25.000 kilos de aguacate hass, ¿cuál será el valor de la maquila?

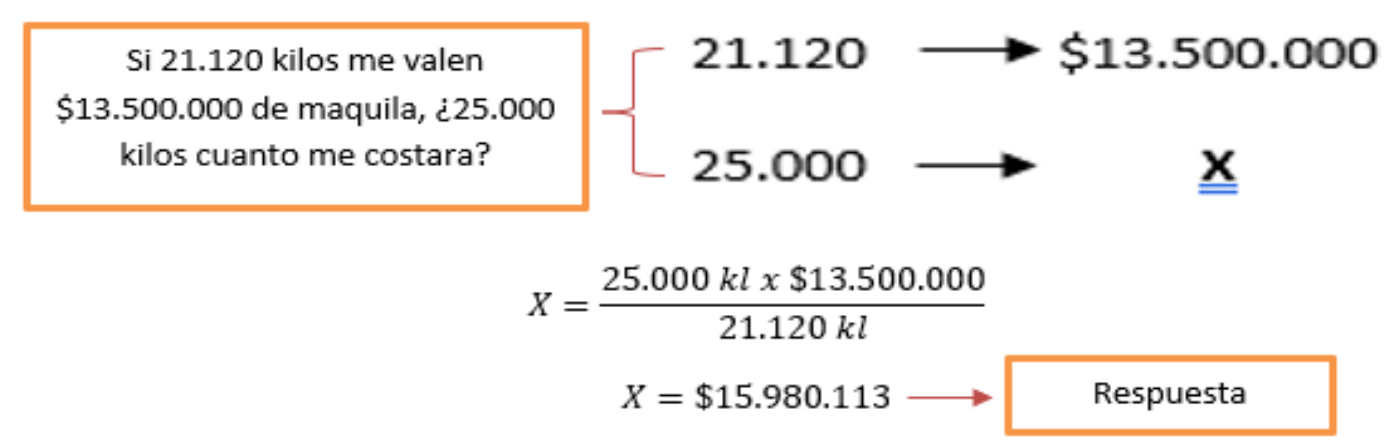

$\mathbf{X}=$ Ira en la columna de Maquila (si los kilos no varían ira la base de la maquila $\$ 13.500 .000)$

Kilos $=$ Ira en Kilos exportados

- Otra columna se denomina "valor a terceros", la cual también tiene una hoja adicional en el Excel con el mismo nombre donde se describe los gastos en los que se incurrió con el contenedor desde la planta hasta el punto de embarque. Ilustración 6.

Estos valores son reales, por cada contenedor se generan las facturas emitidas por cada tercero, las cuales tienen que archivarse y adjuntarse como soporte en cada una de las liquidaciones.

- La otra columna es denominada "Valor Materiales" que son los mismos insumos que se utilizan en cada envió de contenedor. Primero La empresa determino que insumos se necesitan para un pallet (cada contenedor posee 20 pallet). Dependiendo los kilos a exportar se saca la relación de insumos, debido que en unos se pueden ir mas materiales que en otros. Ilustración 4. 
- La última casilla de esta sección de costos esta la denominada "Resultado" que es el rendimiento de la operación, en esta parte se procede a tomar el valor en pesos del reintegro y se le resta cada uno de estos costos, si el resultado es negativo entonces no se cobra la administración del $4 \%$ (manejo de la operación). Después de obtener este valor, se procede a repartir a cada uno de los socios, la cual es la cuarta sección.

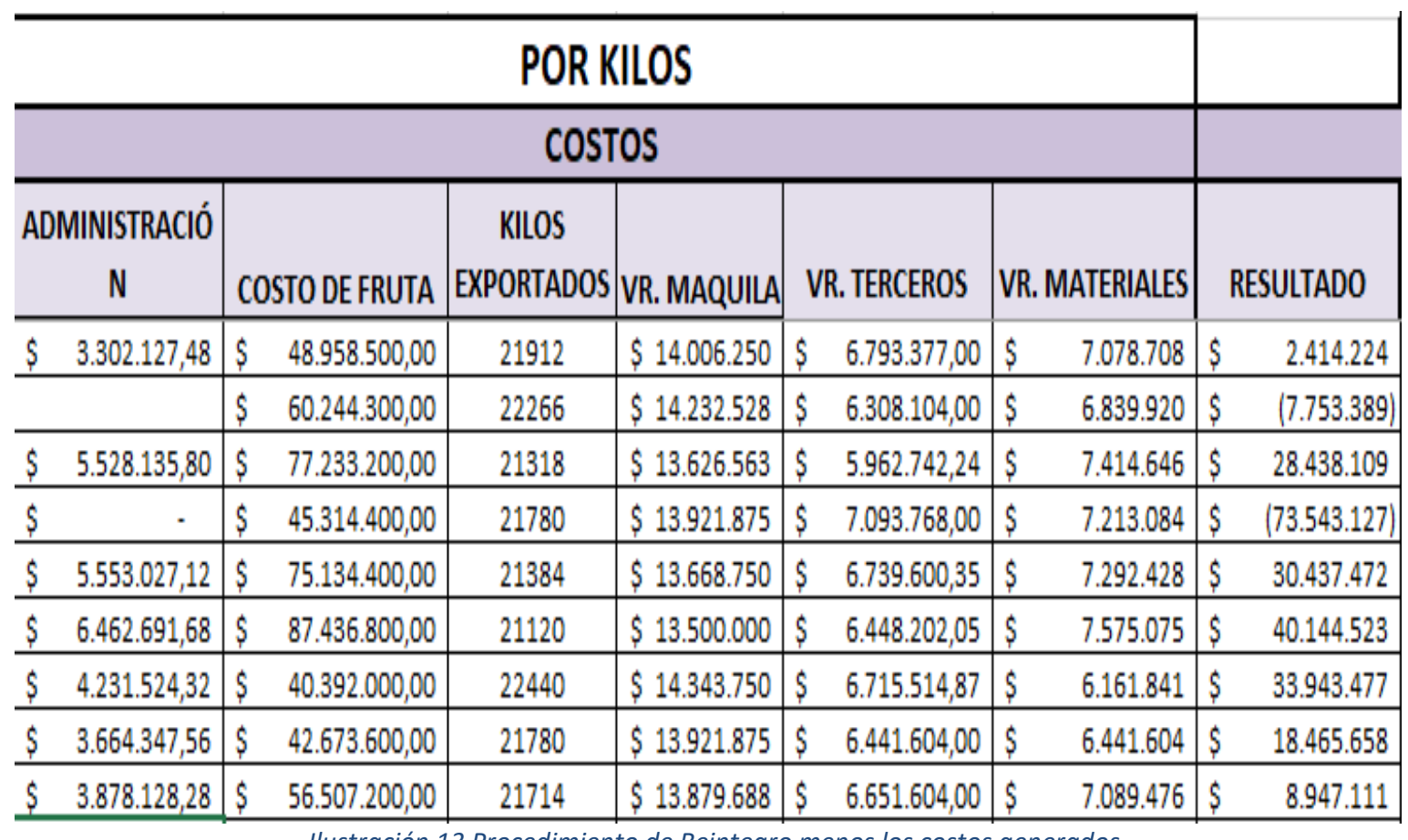

Ilustración 13 Procedimiento de Reintegro menos los costos generados

4. "Participación de los Socios", esta sección se encuentra distribuida por cada uno de los socios incluyendo sus predios, para determinar este proceso, también se tiene una hoja de Excel donde se determina que participación Tuvo cada socio (utilidad o perdida) en él envió, la hoja de cálculo que es el soporte y va unida a esta sección se lleva el mismo nombre de esta sección.

El procedimiento de este es el siguiente:

- En el titulo va el número de la factura que estamos liquidando

- Abajo va el pago del cliente en pesos (se arrastra de la primer parte del cuadro de la columna denominada "reintegro en pesos"). 
- Se verifica con el packing list cuantos de los predios descritos en este pertenecen a los socios, de igual manera solo es para tenerlo en cuenta para el último paso de este, ya que el procedimiento a realizar se hace con todos los predios del packing.

- Se hace un primer cuadro informativo (por packing), el cual va a contener el calibre que se exporto según la factura de venta y el valor que pago el cliente por cada calibre, también el predio y las cajas que vendió cada predio (la información de las cajas se saca del primer paso que corresponde al costo por fruta, en la liquidación el packing están las cajas por calibre).

- Se totalizan las cajas y se comparan con las cajas exportadas descritas en la factura de venta para corroborar que la información este bien.

- Multiplicamos el valor que el cliente pago el calibre por el total de cajas, obteniendo como resultado el total que el cliente pago a cada predio por las cajas que envió según su calibre

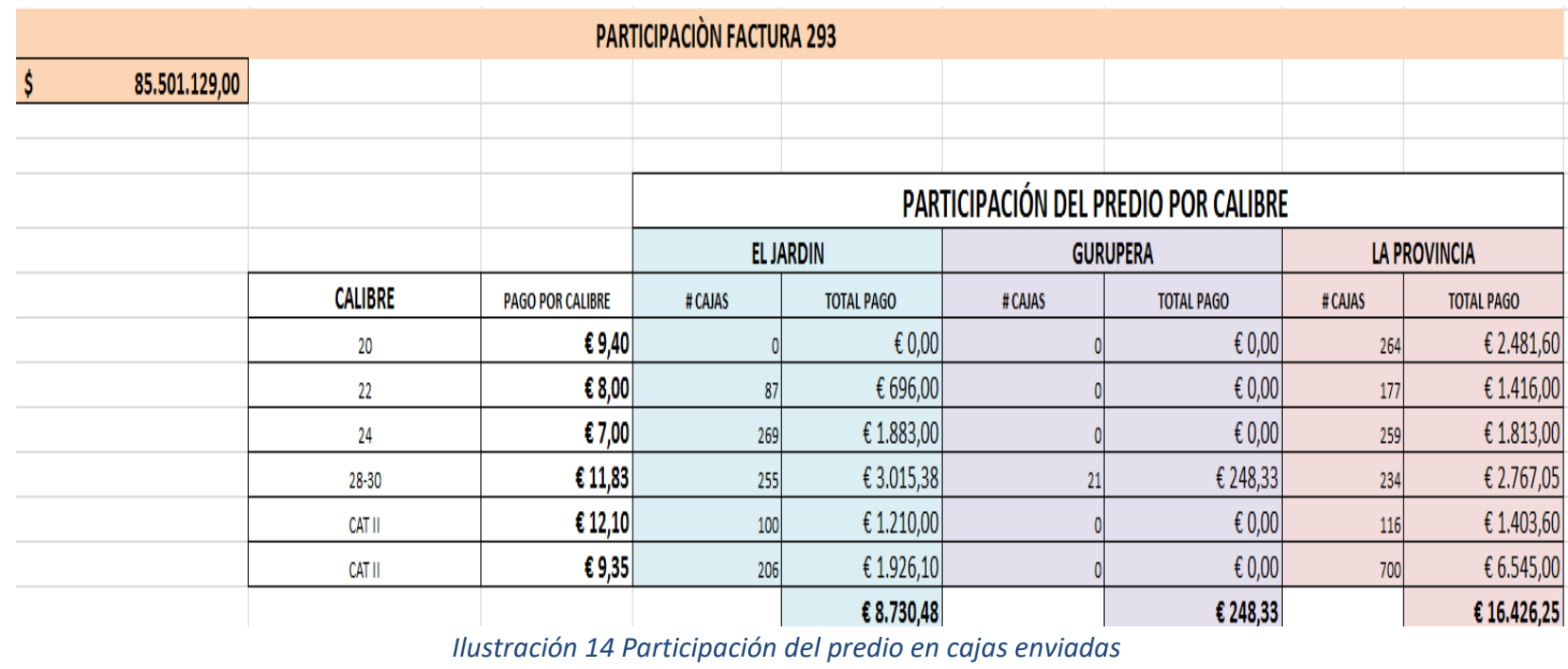

- Se procede a realizar el segundo cuadro que va unido al anterior, en esta ira el nombre de cada predio, el ítem de cada factura: número de cajas por calibre, el pago por cada calibre, el pago total por ítem con este último se saca el porcentaje de cada ítem. 
- Cada predio tendrá el porcentaje de participación en cada ítem el cual se halla teniendo en cuenta el porcentaje de cada ítem por medio de una regla de 3.

- El total de pago correspondiente a este cuadro es el total que recibió cada predio (pago del cliente) por su participación en cada calibre. En el total de pago, se toma el valor que está abajo del número de factura (el valor tomado del reintegro) y se multiplica por el porcentaje de participación de este.

- Se procede a sumar el porcentaje por de cada predio y ese es el porcentaje global de cada predio (la participación que tuvo en ese contenedor). Ese porcentaje es el que tomaremos para finalizar el cuadro.

\begin{tabular}{|c|c|c|c|c|c|c|c|c|c|c|}
\hline \multirow[b]{2}{*}{ TOTAL PAGADO } & \multicolumn{10}{|c|}{ PARTICPACIÒN FACTURA 293} \\
\hline & $\$$ & $120,000,000.00$ & & & & & & & & \\
\hline & & & \multirow[b]{3}{*}{ CALIBRE } & \multirow[b]{3}{*}{ PAGOPORCAUBRE } & \multicolumn{6}{|c|}{ PARTICIPACIÓN DEL PREDIO POR CALLBRE } \\
\hline & & & & & \multicolumn{2}{|c|}{ El JARDIN } & \multicolumn{2}{|c|}{ GURUPERA } & \multicolumn{2}{|c|}{ LAPROVINCIA } \\
\hline & & & & & iccuAs & TOTALPAGO & ICANAS & TOTALPAGO & ICANS & TOTALPAGO \\
\hline & & & 20 & 69.40 & 0 & 60.00 & 0 & 60.00 & 264 & $62,481.60$ \\
\hline & & & 22 & 68.00 & 87 & 0696.00 & 0 & 60.00 & 177 & $(1,416.00$ \\
\hline & & & 24 & 67.00 & 269 & $61,883.00$ & 0 & 60.00 & 259 & $61,813.00$ \\
\hline & & & 28.30 & (11.83 & 255 & $(3,015.38$ & 21 & 6248.33 & 234 & $(2,767,05$ \\
\hline & & & carli & $€ 12.10$ & 100 & $(1,210,00$ & 0 & 60.00 & 116 & $61,403.60$ \\
\hline & & & atil & 69.35 & 206 & $61,926.10$ & 0 & 60.00 & 700 & $66,545.00$ \\
\hline & & & & & & $(8,730.48$ & & 6248.33 & & $\{16,426.25$ \\
\hline
\end{tabular}

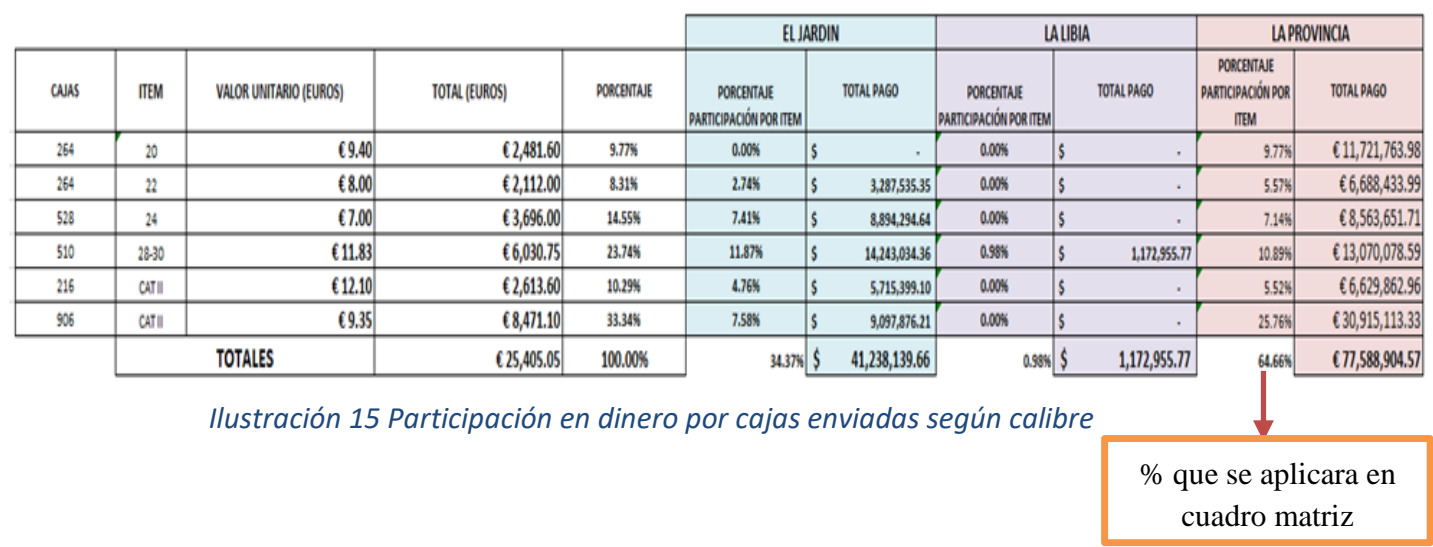


Volviendo al cuadro matriz, y en su parte final, se debe tener en cuenta solo los predios que pertenecen a los socios por cada factura, el "resultado" a la tercera parte de este cuadro, (sea utilidad o perdida) se multiplica por el porcentaje hallado anteriormente por predio. Esta será la participación de cada socio en dinero por contenedor.

\begin{tabular}{|c|c|c|c|c|c|c|c|c|c|c|c|c|c|}
\hline \multicolumn{14}{|c|}{$\begin{array}{c}\text { Valor base para aplicar } \\
\text { porcentaje }\end{array}$} \\
\hline \multirow{3}{*}{\multicolumn{2}{|c|}{$\uparrow$}} & \multirow{2}{*}{\multicolumn{12}{|c|}{ POR DINERO }} \\
\hline & & & & & & & & & & & & & \\
\hline & & \multicolumn{12}{|c|}{ PARTICIPACIÓN SOCIOS } \\
\hline \multirow{3}{*}{\multicolumn{2}{|c|}{$\begin{array}{l}\text { nesuttadoo } \\
\text { S }\end{array}$}} & \multicolumn{2}{|c|}{ DORANCE FLOREZ } & \multirow{2}{*}{\multicolumn{2}{|c|}{$\begin{array}{l}\text { EDILSON } \\
\text { ZAPATA (LA } \\
\text { UBLA) }\end{array}$}} & \multirow[b]{2}{*}{$\begin{array}{c}\text { RICARDO VALENCIA } \\
\text { (EL IAEDIN) }\end{array}$} & \multirow{2}{*}{\multicolumn{2}{|c|}{\begin{tabular}{|c|} 
JOAGE \\
CAMPUEANO \\
(PRIMANERA)
\end{tabular}}} & \multirow[b]{2}{*}{$\begin{array}{c}\text { OSCAR GONEZ (LA } \\
\text { REGAOA) }\end{array}$} & \multirow[b]{2}{*}{$\begin{array}{c}\text { MEUNNA } \\
\text { (AGRICOLA WTA) }\end{array}$} & \multicolumn{3}{|c|}{ GLORIA SANIN } \\
\hline & & LOS NARANUOS & LOS ESPARRAGOS & & & & & & & & LAS ACACINS & COREUNA & MONTERA \\
\hline & & $\$(10,077,764.32)$ & s & $\$$ & $\cdot$ & s & $s$ & - & \$ $(1,469,760.54)$ & s & $s$ & \$ & s \\
\hline & $\$ \quad 8,670,062.88$ & $\$ \quad-1$ & s & \$ & - & $\$$ & $\$$ & - & $\$ \quad 658,924.78$ & $\$$ & $\$$ & \$ $5,505,489.93$ & $\$$ \\
\hline$\$$ & $\$ 28,833,613.08$ & $\$$ & $\$$ & $\$$ & - & $\$ 3,258,198.28$ & $\$$ & - & $\$ \quad 5,112,199,60$ & $\$$ & $\$$ & \$ $3,413,899.79$ & s \\
\hline$\$$ & $\$ 16,749,562.88$ & 200.994 .75 & $\$$ & $\$$ & 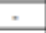 & $\$$ & $\$$ & $=$ & \$ $1,055,222,46$ & $\$$ & $\$$ & $\$ 15,476,596.10$ & s \\
\hline & $\$ 45,055,894,72$ & $\begin{array}{|lr|}\$ & 6,654,755.65 \\
\end{array}$ & $\$$ & s &. & $s$ & s & 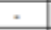 & $\begin{array}{|ll|}\text { S } & 16,661,669.87 \\
\end{array}$ & s & $s$ & $\$ 10,477,495.52$ & s \\
\hline$\$$ & $\$ 24,373,290,72$ & $\$$ & $\$$ & $\$$ & - & $\$$ & $\$$ & - & \begin{tabular}{|l|l|} 
& $5,169,574.96$ \\
\end{tabular} & S $5,908,085,67$ & S $2,437,329.07$ & $\$$ & \$ $2,437,329,07$ \\
\hline$\$$ & $\$ 35,698,838.20$ & $\$$ & $\$$ & $\$$ & - & $\$$ & $\$$ & - & $\$$ & $\$$ & \$ $18,574,884.86$ & \$ $6,707,821.09$ & $\$$ \\
\hline & $\$(82,569,300.00)$ & $\$$ & $\$$ & $\$$ & . & $\$$ & $\$$ & $\cdot$ & $\$ \quad(561,471.24)$ & $\$(4,128,465.00)$ & $\$ \quad[817,436.07)$ & $\$$ & $\$[2,534,877.51$ \\
\hline$s$ & $\$ 18,404,140.80$ & $s$ & $\$$ & $\$$ & . & s & $s$ & 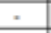 & s & S $3,662,424,02$ & S $1,748,393.38$ & $92,020.70$ & \$ $1,877,222.36$ \\
\hline 5 & S $21,350,518.72$ & $\$$ & $\$$ & $\$$ & - & $s$ & $s$ & - & $\$$ & $s$ & S $12,810,311.23$ & $\$$ & S $8,540,207,49$ \\
\hline$\$$ & $\$ 25,690,446,08$ & $\$$ & $\$$ & $\$$ & - & $\$$ & $\$$ & - & $\$$ & \$ $8,991,656.13$ & S $2,569,044.61$ & $\$$ & \$ $5,138,089.22$ \\
\hline$\$$ & $\$(38,807,045.00)$ & $\$$ & $\$$ & $\$$ & 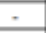 & $\$$ & $\$$ & - & $\$(11,409,271.23)$ & $\$$ & $\$(3.850,704.50)$ & $\$$ & $\$$ \\
\hline$\$$ & $\$ 61,181,902.60$ & $\$$ & $\$$ & $\$$ & $\cdot$ & $\$$ & $\$$ & 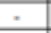 & \$ $14,683,656.62$ & $s$ & \$ $45,274,607.92$ & $\$$ & s \\
\hline$\$$ & $\$ \quad 59,607,553.92$ & $\$$ & s & $\$$ & 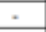 & s & $\$$ & - & $\begin{array}{|ll|}\text { S } & 5,960,755.39 \\
\end{array}$ & $s$ & S $26,823,399.26$ & $\$$ & $\$$ \\
\hline$\$$ & 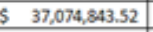 & $\$$ & $\$$ & $\$$ & - & $\$$ & $\$$ & - & $\$ \quad 3,707,484,35$ & $\$ 22,244,906.11$ & S $9,268,710.88$ & $\$$ & $\$$ \\
\hline$\$$ & $\$ 19,724,346.56$ & $\$$ & $\$$ & $\$$ & - & 5 & $\$$ & - & $\$$ & $\$$ & \$ $19,724,346.56$ & $\$$ & $s$ \\
\hline$\$$ & $\$ 23,426,218.40$ & $\$$ & $\$$ & $\$$ & 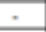 & $\$$ & $\$$ & $=$ & \$ $4,685,243.68$ & \$ $1,171,310.92$ & \$ $7,027,865.52$ & $\$$ & $\$$ \\
\hline s & $\$ \quad 51,727,408.96$ & $\$$ & $\$$ & $\$$ & $\cdot$ & s & $\$$ & $=$ & \$ $15,518,222.69$ & $s$ & S $21,208,237.67$ & s & s \\
\hline$s$ & $s \quad(30,225,821.00)$ & $\$$ & $\$$ & $\$$ & $\cdot$ & 5 & $\$$ & $\cdot$ & $\$(16,926,459.76]$ & $s$ & S $[3,022,582,10)$ & s & 5 \\
\hline
\end{tabular}




\section{Resultado del Proyecto}

Después de haber implementado hace 4 meses este proyecto en la empresa, se ha podido dar claridad a cada uno de los socios de lo que pasa con los contenedores exportados y la participación que han tenido en cada envió (como dueños de los predios). Se espera seguir implementando y alimentando el cuadro a medida que se vayan creando facturas de exportación.

Dichas reliquidaciones se pagaron en el mes de noviembre de 2017 a cada socio, según su participación en el año.

\section{Conclusiones}

Se tiene un gran control en cada factura de exportación puesto que aparte de informa a los socios de su participación ayuda a tener control, ya que se convierte en un estado de cartera de estas, trae expreso la utilidad o la perdida adquirida, y esto es bueno ya que si es perdida se analiza minuciosamente para saber que está pasando, con el fin de da una mejora a los dichos errores cometidos para futuras exportaciones. 


\section{Glosario}

- Calibre: Medida del aguacate según su tamaño. Viene del cal 12/ cal 14 / cal 16 / cal 18 / cal 20 / cal 22 / cal 24 / cal 26 / cal 28-30 y CAT II, siendo cal 12 el más grande y sano y CATII siendo el no exportable y el cual se vende en el mercado nacional.

- Trazabilidad: Es un número que identifica el ingreso de la fruta, este número contiene 11 dígitos descritos de la siguiente manera: Cada predio tiene un numero de 4 dígitos (ningún predio es igual a otro) + la fecha de ingreso por día, mes y año. Este número identificara la fruta desde el momento de ingreso hasta el momento de llegada a destino. Todo con la finalidad que si hay un problema con la fruta al momento en que llega al cliente (que llegue con residualidad o que llegue en mal estado), podamos analizar la fruta

- Residuaidad: Que el aguacate contenga cualquier residuo de plaguicidas (venenos usados por los agricultores para prevenir plagas o enfermedades en aguacates), lo que puede causar el daño del aguacate o simplemente un daño para el ser humano, por ende, se procede a quemar la mercancía ya que no se podrá vender en el país de destino.

- Rendimiento: Es el término utilizado después de maquilar fruta, ya que después de la maquilada queda una cantidad exportable y otra denominada como descarte que es el aguacate que no cumple los requisitos para exportar; después de maquilar la fruta queda seleccionada por calibre y dependiendo del calibre será el valor que se pagará, cuando ya todo esto este hecho recibe el nombre de "rendimiento" de la fruta. 


\section{Referencias}

- Pagina web empresa Tropy fruits S A S (web: http://tropyfruits.com.co/.2013)

- $(\underline{R E F}$. http://www.asohofrucol.com.co/interna.php?cat=4\&scat=27\&act=1

- $\quad$ REF. http://actualicese.com/2016/10/04/compra-de-productos-agricolas-opecuarios-y-sus-tarifas-de-retencion-en-la-fuente/)

- REF. Presentación powerPoint/ CORPOHASS - El Gremiod de la Agroindustria de Aguacates Hass). 